\title{
AN INEQUALITY FOR THE BINARY ENTROPY FUNCTION AND AN APPLICATION TO BINOMIAL COEFFICIENTS
}

\author{
VANIA MASCIONI
}

Abstract. We prove a tight inequality of non-exponential type for a weighted geometric mean commonly appearing when using Stirling's approximation (also frequently studied in its logarithmic form when computing entropies). As an application we prove corollaries involving binomial coefficients.

Mathematics subject classification (2010): Primary: 26E60, Secondary: 26D15,05A10, 26D20.

Keywords and phrases: Weighted geometric mean, binary entropy function, Stirling approximation, binomial coefficient.

\section{REFERENCES}

[1] D. S. Mitrinović, Analytic Inequalities, Springer-Verlag, 1970.

[2] D. J. C. MACKAY, Information Theory, Inference, and Learning Algorithms, Cambridge University Press, 2003.

[3] H. RobiIns, A remark on Stirling's formula, Amer. Math. Monthly 62 (1955), 26-29.

[4] P. STǍNICǍ, Good lower and upper bounds on binomial coefficients, Journal of Inequalities in Pure and Appl. Math. 2 (2001), Issue 3, Article 30 (http://www .emis.de/journals/JIPAM/). 